\title{
QUANTITATIVE PROTEOME AND ACIDIC SUBPROTEOME PROFILING OF CANDIDA ALBICANS YEAST-TO-HYPHA TRANSITION
}

\author{
L. Monteoliva ${ }^{(1)}$, R. Martínez- López ${ }^{(1)}$, A. Pitarch $^{(1)}$, M.L. Hernáez $^{(2)}$, A. Serna $^{(2)}$, C.
} Nombela $^{(1)}$, J.P. Albar $^{(3)}$, C. Gil ${ }^{(1)}$.

(1) Dpto. Microbiología II. Universidad Complutense de Madrid, ${ }^{(2)}$ Servicio de Proteómica, UCM-Parque Científico de Madrid, Spain, ${ }^{(3)}$ Servicio de Proteómica, Centro Nacional de Biotecnología, CSIC, Madrid, Spain.

Candida albicans yeast-to-hypha morphological transition is involved in the virulence strategy of this opportunistic fungal pathogen. Changes in relative abundance of the Candida proteome related to this process were analysed using different twodimensional differential in-gel electrophoresis (2D-DIGE)-based approaches. First, a comparative analysis of yeast and hyphal cytoplasmic proteins allowed the detection of 106 protein spots with significant variation in abundance. Sixty-one of them, corresponding to 46 proteins, were identified. As most of the differentially abundant proteins had an acidic isoelectric point, a large-scale pre-fractionation approach to analyse the acidic C.albicans subproteome was carried out. Ninety acidic C. albicans proteins were identified by either gel-based or non-gel-based approaches. Additionally, different workflows combining preparative isoelectric focusing, Cy labelling and narrow $\mathrm{pH}$ gradient 2-DE gels were tested to analyse the differences in relative protein abundance between yeast and hyphal acidic subproteomes. It was possible to identify 21 differentially abundant acidic proteins, 10 of them were not identified in the previous 2D-DIGE gels. Functional and network interaction analyses of the 56 differentially abundant proteins identified by both approaches rendered an integrated view of metabolic and cellular process reorganization during the yeast-to-hypha transition. With these results, we propose a model of metabolic reorganization. 